\title{
Amplitude and wall-normal distance variation of small scales in turbulent boundary layers
}

\author{
Theresa Saxton-Fox $\odot,{ }^{1, *}$ Adrián Lozano-Durán ${ }^{0},{ }^{2}$ and Beverley J. McKeon $\circledast^{1}$ \\ ${ }^{1}$ Department of Mechanical and Civil Engineering, California Institute of Technology, \\ Pasadena, California 91125, USA \\ ${ }^{2}$ Department of Aeronautics and Astronautics, Massachusetts Institute of Technology, \\ Cambridge, Massachusetts 02139-3291, USA
}

(Received 7 October 2021; accepted 3 January 2022; published 20 January 2022)

The spatial organization of small scales around large-scale coherent structures in a flat plate turbulent boundary layer is studied using a conditional-averaging technique applied to experimental and computational data. The technique averages the small-scale velocity conditioned on the projection coefficient between the instantaneous streamwise velocity field and a model for large-scale velocity structures in the wake and logarithmic regions. Two distinct scenarios are identified for the organization of the small scales: amplitude variation, in which at a given wall-normal location the small-scale intensity varies in amplitude across the streamwise extent of the large-scale structure, and height variation, in which the small-scale velocity intensity remains nearly constant along a curve that changed its wall-normal location across the streamwise extent of the large-scale structure. Small scales that are energetic at the wall-normal location where the large-scale structure is centered primarily show evidence of height variation, while small scales that are energetic at wall-normal locations far from the center of the large-scale structure primarily show evidence of amplitude variation. Connections can be drawn between the statistical observations characterized by the amplitude modulation statistic and the structural picture associated with vortex clusters.

DOI: 10.1103/PhysRevFluids.7.014606

\section{INTRODUCTION}

Coherent structures populate turbulent boundary layers and are central to their chaotic dynamics [1]. These structures can be separated into large and small features through comparison to a reference length scale. Using a reference length scale on the order of the boundary layer thickness, the large-scale coherent structures are the very-large-scale motions (VLSMs) or superstructures [2-4] in the logarithmic region and the large-scale motions (LSMs) in the wake region $[5,6]$. The most commonly discussed small-scale coherent structures are the streamwise streaks [7] in the near-wall region and vortices (hairpin, cane, horseshoe, "typical eddies," vortex clusters, etc.) in the logarithmic and wake regions $[4,5,8,9]$. Very-large-scale motions and LSMs appear instantaneously (without the mean subtracted) as bulges in the streamwise velocity field, with regions of uniform momentum in their centers bounded by shear layers [10-13]. Small-scale vortices have been observed in shear layers along the backs of low-speed streamwise velocity regions [5,9]. These vortices may have a hairpin vortex structure: thin and long, arching over large scales in the flow $[5,8]$, though the persistence of hairpin vortices at high Reynolds number is a topic of continued

\footnotetext{
*Present address: Department of Aerospace Engineering, University of Illinois at Urbana-Champaign, Urbana, Illinois 61801, USA, tsaxtonf@illinois.edu
} 


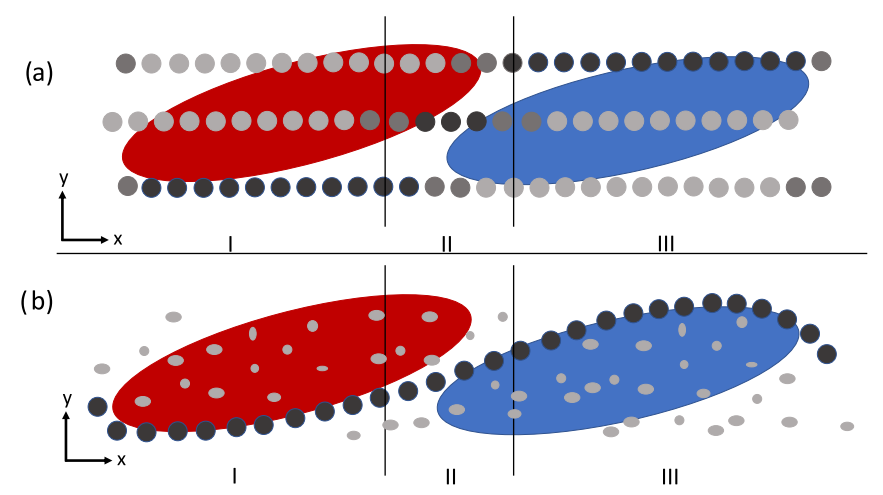

FIG. 1. Schematic representations of (a) amplitude variation and (b) height variation. Large-scale fluctuating streamwise velocity structures are shown in red (fast) and blue (slow), while small scales are shown in gray (dark indicating strong amplitude and light indicating weak amplitude).

debate $[4,14,15]$. The vortices may also have a more disorganized or chaotic structure at higher Reynolds number [4].

The interaction of coherent structures enables the exchange of energy between scales and sustains the turbulent state [16]. Preferential organization of one structure depending on the behavior of another structure is one indication of interaction. Small-scale vortices at the edge of the boundary layer and near the wall are organized around and influenced by large-scale velocity motions $[9,17,18]$. The organization between large and small scales in turbulent boundary layers has been quantified by filtering the velocity field into large and small scales and correlating the filtered signals, using data from hot-wire measurements [19], large-eddy simulation [20], and direct numerical simulation [21]. In each case, the envelope and intensity of the small scales was observed to be strongly correlated to the large-scale streamwise velocity signal with the sign of correlation changing from positive near the wall to negative farther away. This relationship was found to be robust across Reynolds numbers [22], geometries [23], pressure gradients [24], small-scale velocity components [25], and wall permeability [26].

The observed correlation between large and small scales permits several interpretations of the instantaneous organization of large and small coherent structures, including amplitude variation and height variation. We focus here on these two instantaneous interpretations, which are illustrated in Fig. 1 with flow moving from left to right. Large-scale fluctuating coherent structures of streamwise velocity are shown in red (positive) and blue (negative) and small-scale coherent structures are shown as circles with low amplitude (light gray) and high amplitude (dark gray). In the case of amplitude variation [Fig. 1(a)], multiple small-scale coherent structures, each at a fixed height, change amplitude depending on the local behavior of the large-scale coherent structure. This is the stress organization often inferred from the spectral picture offered by traditional conditional averaging (see, e.g., [20,27]). In the case of height variation [Fig. 1(b)], a single small-scale coherent structure with a fixed amplitude changes height depending on the local behavior of the large-scale coherent structure, while weak, incoherent small-scale activity is present throughout the flow. Height variation is aligned with preferential vortex alignment on the back of low-speed structures (see, e.g., $[5,17]$ ) and with the ejection, sweep, and burst representation of the flow (see, e.g., [18]). The amplitude variation and height variation representations lead to similar statistics but imply different mechanisms driving the organization.

The schematic in Fig. 1 is broken up into three regions in the streamwise direction to examine the local behavior. In Figs. 1(a) and 1(b), in region I the large-scale streamwise velocity fluctuation is positive and small scales are strongest near the wall; in region II, the large-scale transitions from positive to negative and small scales are strongest at a middle height; and in region III, the 
large scale is negative and small scales are strongest away from the wall. The two explanations differ in the spatial organization of the small-scale coherent structures and in the driving scale organization mechanism that they suggest. Amplitude variation may point to an energy transfer or stress-based mechanism, while height variation may point to a convective mechanism. The two behaviors are visually distinguishable by the continuity of the small-scale intensity across regions I-III. In amplitude variation [Fig. 1(a)], a given small-scale coherent structure appears continuously at a fixed height across the three regions. In height variation, a given small-scale coherent structure appears continuously along a curve of changing height across regions I-III. The present work aims to quantify the small-scale intensity across the streamwise extent of a large-scale coherent structure to investigate whether amplitude or height variation is dominant.

The present study uses spatially resolved measurements and a different conditional averaging technique to study the averaged small-scale velocity intensity across a continuous range of phases of large-scale coherent structures. Both amplitude and height variation are observed with different levels of importance depending on the type of large scale being averaged upon and the component of the small-scale velocity intensity being considered. Spatial data are obtained using particle image velocimetry and direct numerical simulation. A global conditional averaging technique, termed conditional projection averaging, is used to correlate the large-scale signal and small-scale intensity. The technique yields a smoothly varying, averaged large-scale field and a correlated smoothly varying, averaged small-scale intensity field. The results of the averaging process are provided, including the extent to which height and amplitude variation are observed in each component of the velocity field. The sensitivity of the results to the details of the analysis technique are offered in the Appendix.

\section{SOURCES OF DATA}

Turbulent boundary layer data were obtained from particle image velocimetry (PIV) measurements in an experimental facility from Saxton-Fox [28] and direct numerical simulation (DNS) from Wu et al. [29]. Data extracted from both sources were at similar Reynolds numbers, $\operatorname{Re}_{\theta}=3300$ and $\mathrm{Re}_{\tau}=910$ for the experiment and $\mathrm{Re}_{\theta}=3000$ and $\mathrm{Re}_{\tau}=900$ for the DNS. The experimental data were collected in the Merrill wind tunnel at the California Institute of Technology, an incompressible, recirculating facility. The turbulent boundary layer was developed in a $0.6 \times 0.6 \mathrm{~m}^{2}$ test section with a flat plate that had a wire trip at the leading edge. The velocity was measured in the streamwise-wall-normal plane using a double-pulsed YLF laser and a Photron Fastcam APX-RS camera with a $17-\mathrm{mm}$ Tamron macrolens. The measurement was recorded at a rate of $1.5 \mathrm{kHz}$ with a pulse separation of $35 \mu$ s and yielded data with a field of view of $1.4 \delta \times 1.7 \delta$, where $\delta$ refers to the $99 \%$ boundary layer thickness. DaVis software from LaVision was used to process the data; a double pass approach with windows of first 32 and then 16 pixels with $50 \%$ overlap was used to yield a velocity field with $0.013 \times 0.013$ outer units or $14.5 \times 14.5$ inner units resolution per vector $[28,30]$.

For the computational data, the boundary layer was simulated from its initial laminar state through a bypass transition to a fully canonical turbulent state $[29,31]$. In the present work, the downstream portion of the simulation, where $\operatorname{Re}_{\theta} \approx 3,000$, was analyzed. In this region, the spanwise extent of the simulation was approximately $2.6 \delta$, using the local $99 \%$ boundary layer thickness. Many streamwise-wall-normal planes were extracted from the DNS data at different spanwise locations. These planes were compared directly to the PIV data and improved the convergence of the analysis techniques, as only 30 temporal snapshots were available for the computational data, compared to 5120 temporal snapshots for the experimental data. Converged statistics of the flow were available for comparison to the experimental data just upstream of the region of interest, at $\operatorname{Re}_{\theta}=2$, 900, which are shown in Fig. 2 .

The notation adopted for the coordinate system was $(x, y, z)$ (streamwise, wall-normal, spanwise), with velocity components $\mathbf{U}=(U, V, W)$ in the streamwise, wall-normal, and spanwise directions. The mean velocity field of each data set $\overline{\mathbf{U}}(x, y)$ was computed by performing a 


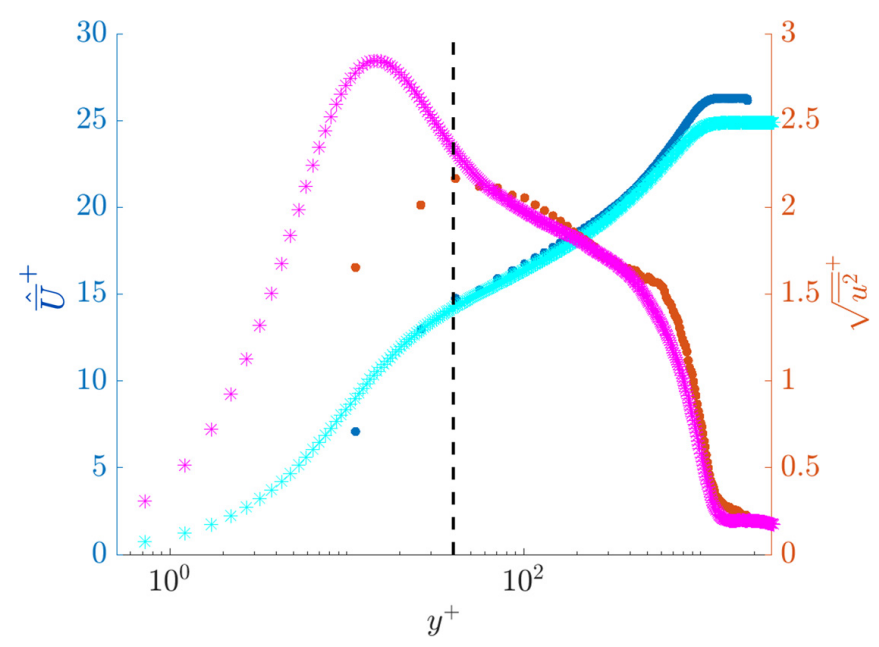

FIG. 2. Mean profile (blue dots) and rms (red dots) for the streamwise velocity from the PIV data at $\operatorname{Re}_{\theta}=$ 3300 as a function of distance from the wall and mean profile (cyan stars) and rms (magenta stars) for the streamwise velocity from the DNS data at $\operatorname{Re}_{\theta}=2900$ [31]. The dashed line indicates the wall-normal distance below which reflections corrupted the experimental data.

temporal average (for the experimental data) and combined temporal and spanwise average (for the DNS data) of the velocity field. The fluctuating velocity about the mean velocity field was defined as $\mathbf{u}(x, y, z, t)=\mathbf{U}(x, y, z, t)-\overline{\mathbf{U}}(x, y)$. A characteristic mean velocity profile $\hat{\bar{U}}(y)$ was defined for the experimental data by performing a temporal average for the experimental data at a streamwise location in the center of the field of view. The computational mean velocity profile had been computed in the original computational campaign just upstream of the region of interest, at $\operatorname{Re}_{\theta}=2$, 900. Similarly, the root mean square streamwise velocity fluctuation was calculated in the center of the field of view for the experimental data (Fig. 2). The data were normalized using viscous units, with length scales normalized by $v / u_{\tau}$ and velocities normalized by $u_{\tau}$, where $v$ is the kinematic viscosity and $u_{\tau}$ is the friction velocity. The friction velocity was estimated for the experimental data using the Clauser method [32,33]. When compared to data from Wu et al. [31] at similar Reynolds numbers, the statistics of the experimental data were found to agree fairly well above $y^{+} \approx 40$ or equivalently $y / \delta \approx 0.05$. The statistics were also separately compared to those from DeGraaff and Eaton [34] (not shown here) and showed agreement above $y^{+} \approx 40$ or equivalently $y / \delta \approx 0.05$. Below $y^{+} \approx 40$, reflections from the laser impingement on the flat plate caused inaccuracies in the velocity measurement.

Both experimental and computational data were used in the analysis that follows. The use of experimental or computational data was not found to alter the trends observed in the results, and therefore each result was shown using either experimental or computational data. The experimental data had the benefit of a larger number of temporal snapshots available for analysis, which provided additional confidence in the convergence of the results. The computational data had the benefits of improved spatial resolution, improved accuracy very near the wall, a larger field of view, and access to the spanwise component of velocity. The experimental data were used for streamwise and wall-normal velocity results in the outer and logarithmic regions of the flow, while computational data were used in the very-near-wall region and for the spanwise component of velocity. The number of snapshots used for each result is provided in Table II. To test whether the difference in spatial resolution between the two data sets affected the results, the smallest scales were filtered from the data; the final amplitude of the results varied but the trends did not. 


\section{METHODS}

\section{A. Decomposition of velocity into large and small scales}

A Gaussian filter was used to decompose the turbulent data into large and small scales in the $x$ and $y$ directions $[35,36]$. An approximate cutoff frequency can be defined as the frequency where the filtered signal is half of its maximum in the power spectrum, given by

$$
f_{c}=\frac{\sqrt{2 \ln (2)}}{2 \pi \sigma_{G}},
$$

where $\sigma_{G}$ is the standard deviation of the Gaussian function. The values used for the standard deviation in this study were $\sigma_{G} / \delta=[0.125,0.5]$, corresponding to a range of approximate cutoff wavelengths $\lambda_{G} / \delta=[0.67,2.7]$. These approximate cutoff wavelengths bounded the value used by Mathis et al. [37], $\lambda_{x} / \delta=1$, and were within the range of filter sizes considered by Ganapathisubramani et al. [27]. The final results were shown to be qualitatively insensitive to the filter size in the Appendix.

The fluctuating large-scale streamwise velocity field $u_{L}$ was defined by convolving the Gaussian function with the fluctuating streamwise velocity field. This convolution was normalized by the integral of the filter in the domain to minimize edge effects. The small scales were computed by subtracting the fluctuating large-scale velocity field from the fluctuating velocity field

$$
u_{s}\left(x, y, z_{i}, t_{i}\right)=u\left(x, y, z_{i}, t_{i}\right)-u_{L}\left(x, y, z_{i}, t_{i}\right) .
$$

The equivalent procedure was used to define the large and small scales in the wall-normal and spanwise velocity fields $v_{L}, w_{L}, v_{s}$, and $w_{s}$, respectively. The small-scale velocity intensity was defined as the square of the small-scale velocity. A mean-added, large-scale streamwise velocity field was defined as the sum of the fluctuating large-scale field with the mean

$$
U_{L}\left(x, y, z_{i}, t_{i}\right)=u_{L}\left(x, y, z_{i}, t_{i}\right)+\bar{U}(x, y) .
$$

\section{B. Model for large-scale coherent structures}

In the present work, a spatially periodic model for the large-scale structures is used. Modeling large-scale coherent structures as periodic in the streamwise and spanwise directions and in time is supported by recent work characterizing the periodicity of large-scale velocity structures [38] and the reported ubiquity of the VLSM frequency for amplitude modulation [39]. Periodic models have a clearly defined phase with wavelengths and wave speeds that are known to be energetic in the flow from experimental and computational results. Periodic models were previously shown to capture instantaneously realistic flow phenomena including bulges, uniform momentum zones, and hairpin vortices $[13,40]$. However, we acknowledge that instantaneous turbulent structures are not periodic and that the parallel flow assumption required to use periodic structures in the streamwise direction introduces limitations in the model.

Resolvent analysis of the Navier-Stokes equations was used to generate a template of large-scale turbulent structures [41]. Following the parallel flow assumption, periodicity was assumed in the streamwise direction and Fourier transforms were performed in the temporal, streamwise, and spanwise variables. Given a mean velocity profile $\hat{U}(y)$ and values of the streamwise wave number $k_{x}$, spanwise wave number $k_{z}$, and temporal frequency $\omega$ that are relevant for a given structure of interest, the Navier-Stokes equations can be reformulated into an input-output system such that the Fourier-transformed nonlinear term $\hat{\mathbf{f}}$ is the input to a linear operator $\mathscr{H}$ and the Fourier-transformed velocity and pressure fluctuations $\hat{\mathbf{u}}$ are the output: $\hat{\mathbf{u}}=\mathscr{H} \hat{\mathbf{f}}$. By performing a singular value decomposition on the linear resolvent operator, the most-amplified input and output structures can be identified as the left and right singular vectors, respectively, $\mathscr{H}=\Psi \Sigma \Phi^{-1}$. We use a rank-1 approximation, such that the first left singular vector of the resolvent operator $\psi_{1}(y)$ is used as the representative wall-normal coherence for the structures at a specified wave number [41]. Finally, the 
TABLE I. Comparison between traditional conditional averaging and conditional projection averaging.

\begin{tabular}{lll}
\hline \hline Parameter & \multicolumn{1}{c}{ Traditional } & \multicolumn{1}{c}{ CPA } \\
\hline $\begin{array}{l}\text { Location of data extracted } \\
\text { to evaluate condition }\end{array}$ & Single point & Regions of overlap with model \\
$\begin{array}{l}\text { Comparison value } \\
\text { Comparison operation }\end{array}$ & $\begin{array}{l}\text { Single value } \\
\text { Greater than or less than }\end{array}$ & $\begin{array}{l}\text { Local values of model } \\
\text { Projection coefficient (greater than) } \\
\text { and maximization over phase }\end{array}$ \\
\hline \hline
\end{tabular}

large-scale model is given by the real part of the sum of a left-propagating and right-propagating wave

$$
\tilde{u}(x, y, z, \tilde{\gamma})=\operatorname{Re}\left[\psi_{1}(y)\left(e^{i\left(k_{x} x+k_{z} z-\omega t-\tilde{\gamma}\right)}+e^{i\left(k_{x} x-k_{z} z-\omega t-\tilde{\gamma}\right)}\right)\right],
$$

which represents a downstream-propagating traveling wave where $\tilde{\gamma}$ is an arbitrary phase.

In the wake region of the boundary layer, where large-scale motions are energetically dominant [6], the model was constructed using assumed wavelengths of $\lambda_{x} / \delta=4$ and $\lambda_{z} / \delta=1$, with a convection velocity of $c / U_{\infty}=\left(\omega / k_{x}\right) / U_{\infty}=0.8$. In the logarithmic layer, where VLSMs are energetically dominant, the model assumed wavelengths of $\lambda_{z} / \delta=6$ and $\lambda_{z} / \delta=1$, with a convection velocity of $c / U_{\infty}=\left(\omega / k_{x}\right) / U_{\infty}=0.6$. Modes derived from resolvent analysis have been shown to be maximized at their critical layer [41], the height where their convection velocity matches the local mean velocity. The convection velocity chosen for the large-scale motion model placed the peak amplitude in the wake region, while the convection velocity for the very-large-scale motion placed it in the logarithmic region.

\section{Conditional projection averaging}

We introduce the conditional projection averaging (CPA) method, which is developed to average a field variable conditioned on the instantaneous streamwise location of a particular large-scale coherent structure. Rather than averaging based on a condition evaluated at a single point, a global condition is utilized through the projection between the spatially resolved instantaneous velocity field and the spatial model of the large-scale structure. The projection between the velocity field and the model acts to simultaneously extract data at the appropriate locations in the flow and evaluate whether the velocity satisfies the chosen condition. This method can be used with structural models that have a defined phase or for which a phase can be defined from the mode's convection in space and time. Resolvent analysis, dynamic mode decomposition, spectral proper orthogonal decomposition, and space-time proper orthogonal decomposition all generate modes that could be used for conditional projection averaging [41-44]. In this work the resolvent analysis described in the preceding section is used to define the model. A summary of the similarities and differences of CPA versus traditional conditional averaging is provided in Table I. The schematic representation of the projection method is provided in Fig. 3 .

In traditional conditional averaging, one point in the flow at a time is generally selected to evaluate a condition $\left[u\left(x_{0}, y_{0}, z_{0}\right)\right]$; in a flow with statistically homogeneous or slowly varying streamwise and spanwise directions, this is equivalent to choosing a single height in the flow at which to evaluate a condition. Multiple heights are often studied in sequence by systematically changing the height of the conditional point in the flow. Global conditional averaging techniques instead use information from many points in space and/or time to condition. Variable-interval time averaging is an example of such a technique that uses local averages of turbulence quantities over a region of time or space [45]. The CPA method used here is a global technique in which instantaneous spatial data are projected onto a spatial model of a large-scale structure; the data that overlap with heights where the model has nonzero amplitude are used to evaluate the condition, while data that overlap with heights where the model has zero or near-zero amplitude are masked by the 


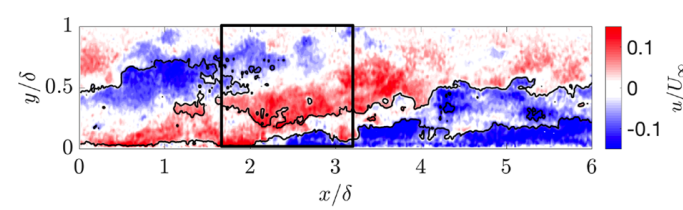

(a)

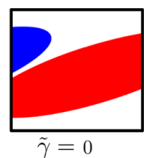

$\tilde{\gamma}=0$

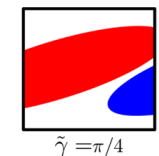

$\gamma=\pi / 4$

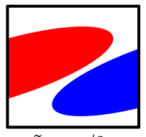

$\tilde{\gamma}=\pi / 2$

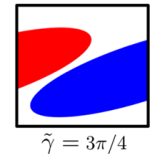

(b)

FIG. 3. Schematic of the projection method between (a) a visualization of the instantaneous fluctuating streamwise velocity field and (b) an illustration of a large-scale model at four locations in its convection, labeled as phases $\tilde{\gamma}$. The black box outlines the approximate size of a single PIV frame. The data are projected onto the large-scale model at each model phase $\tilde{\gamma}$. The value of the projection coefficient is used to evaluate the condition and average together data that compare favorably to the model at the same phase.

multiplication by zero and do not influence the resulting average. The models used in this study have an approximately Gaussian distribution in the wall-normal coordinate with maxima located at their critical layer, i.e., the height where the model's convection velocity matches the local mean speed. The choice of the model's critical layer height (or equivalently convection speed) is analogous to the choice of the wall-normal location in the flow at which the condition is evaluated in traditional conditional averaging.

In traditional conditional averaging, a numeric value is selected with which to compare the instantaneous data at a single point. Conditional projection averaging uses a spatial field rather than pointwise information to evaluate the condition, so a singly valued condition is no longer meaningful. Instead, the data at each spatial point are compared to the value of the model at the same spatial point. The projection operation averages the result of these comparisons together, with larger weight on the agreement in locations with larger model amplitude. In traditional conditional averaging, one often looks at different numeric values for comparison, such as a negative versus positive value. In CPA, one can vary the value of comparison by changing the phase of the periodic model. A shift of $\pi$ yields the binary positive-negative value switch of traditional conditional averaging at any one location, but one can also consider finer phase shifts that change the weighting of different streamwise locations in the computation of the projection coefficient.

In traditional conditional averaging, one determines whether the data satisfy the condition through a greater than or less than operation $\left[u\left(x_{0}, y_{0}, z_{0}\right)\right.$ less than a single value $]$. In CPA, the projection coefficient between the data and model is used to evaluate whether the data satisfy the condition. Two separate operations are used to evaluate the projection coefficient. The first requires that the projection coefficient is greater than some threshold value $R>R_{t h}$, indicating a certain level of average agreement between the data and the model. The second is a maximization condition, which requires that the projection coefficient is larger at the model phase of interest than an an other phase considered. This condition ensures that the instantaneous streamwise position of the large scale in the data is similar to the streamwise position of the large scale in the model. To evaluate the maximization condition, the projection between the data and the model is computed at many model phases. The maximization condition was found to increase the robustness of the method and is schematically demonstrated in Fig. 3.

The projection between the instantaneous fluctuating velocity $u$ and a model $\tilde{u}$ at several model phases $\tilde{\gamma}_{j}$ is given in its most general form as

$$
R\left(t_{i}, \tilde{\gamma}_{j}\right) \equiv\left(\frac{u\left(x, y, z, t_{i}\right) \cdot \tilde{u}\left(x, y, z, \tilde{\gamma}_{j}\right)}{\left|u\left(x, y, z, t_{i}\right)\right|\left|\tilde{u}\left(x, y, z, \tilde{\gamma}_{j}\right)\right|}\right)
$$

where a dot product is performed in the numerator, $t_{i}$ is one snapshot, and $R$ is the projection coefficient. Using the maximization condition, one defines the model phase that yields the highest projection coefficient for a given snapshot,

$$
\gamma\left(t_{i}\right)=\arg \max R\left(t_{i}, \tilde{\gamma}_{j}\right) \forall j .
$$




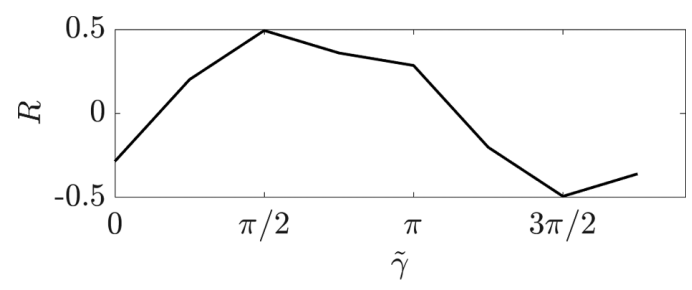

FIG. 4. Projection coefficient $R$ plotted against the model phase $\tilde{\gamma}$ for a single snapshot of experimental data.

This phase represents the approximated large-scale structure phase of the snapshot, $t_{i}$. The process was repeated for each snapshot such that each snapshot was associated with one phase. The threshold condition

$$
R\left(t_{i}, \gamma\left(t_{i}\right)\right)>R_{t h}
$$

enforces that the best projection coefficient has to be larger than a threshold value $R_{t h}$ for that snapshot to be considered in any average based upon the approximated large-scale structure phase.

The two-dimensional projection between the data and the model was defined as

$$
R\left(t_{i}, z_{i}, \tilde{\gamma}_{j}\right) \equiv\left(\frac{u\left(x, y, z_{i}, t_{i}\right) \cdot \tilde{u}\left(x, y, z_{0}, \tilde{\gamma}_{j}\right)}{\left|u\left(x, y, z_{i}, t_{i}\right)\right|\left|\tilde{u}\left(x, y, z_{0}, \tilde{\gamma}_{j}\right)\right|}\right)
$$

where $z_{i}$ is a single spanwise location in the data and $z_{0}$ is a single spanwise location in the model. For the experimental data, only a single spanwise location was available due to the planar nature of the PIV system, while for the computational data, multiple spanwise locations were considered in series. When analyzing the DNS data, a modification was leveraged to improve the efficiency of the computation. The projection operation weights the data-to-model comparison largest at locations where the model has the largest amplitude. The models used in this study have a roughly Gaussian distribution in $y$, with a maximum at their center and a periodic distribution in $z$. To improve efficiency, a one-dimensional projection was used, in which data from each snapshot at each spanwise location $z_{i}$ and at a single wall-normal location $y_{0}$ was projected onto information from the model at each phase $\tilde{\gamma}_{j}$ and at a spanwise location $z_{0}$ and wall-normal location $y_{0}$ where the model was maximized in space. The projection was computed using Eq. (8) with $y_{0}$ substituted for $y$, yielding a one-dimensional projection. The wall-normal location $y_{0}$ for the comparison was the critical layer height of the model. Both one-dimensional and two-dimensional projections were tested on the experimental data; the results are shown in the Appendix to be fairly insensitive to the dimensionality of the projection. Figure 4 shows an example of the variation of $R$ with $\tilde{\gamma}$ for $\tilde{\gamma}_{j}=[0, \pi / 4,7 \pi / 4]$ for a single snapshot of experimental data, $u\left(x, y, t_{e x}\right)$. The approximated large-scale structure phase for the snapshot used in Fig. 4 was $\gamma\left(t_{e x}\right)=\pi / 2$.

Once each snapshot was labeled with a large-scale phase, various flow quantities $q$ from twodimensional planes with the same approximated large-scale phase $\gamma$ were averaged together,

$$
\langle q\rangle(x, y, \gamma)=\frac{1}{N} \sum_{t_{i}} \sum_{z_{i}} q\left(x, y, z_{i}, t_{i}\right) \forall t_{i}, z_{i} \text { s.t. } R\left(z_{i}, t_{i}, \gamma\right)>R\left(z_{i}, t_{i}, \tilde{\gamma}_{j} \neq \gamma\right), \quad R\left(z_{i}, t_{i}, \gamma\right)>R_{t h},
$$

where $N$ is the total number of planes considered for the average. For ease of visual inspection, the averages are organized sequentially in the final results such that the phase continuously varies across the horizontal axis. The number of frames used for the average at each phase condition for each structure and type of data is provided in Table II. 
TABLE II. Number of planes of data used for the averaging in Figs. 6-8. The number of distinct temporal snapshots $N_{t}$ versus total planes including distinct times and spanwise coordinates $N_{t, z}$ are provided. The number of two-dimensional planes per phase bin is also listed.

\begin{tabular}{lrrrrrrrrrr}
\hline \hline$N$ frames & \multicolumn{1}{c}{$N_{t}$} & \multicolumn{1}{c}{$N_{t, z}$} & \multicolumn{1}{c}{0} & $\pi / 4$ & $\pi / 2$ & $3 \pi / 4$ & $\pi$ & $5 \pi / 4$ & $3 \pi / 2$ & $7 \pi / 4$ \\
\hline LSM, PIV & 4385 & 4385 & 481 & 691 & 533 & 399 & 495 & 730 & 650 & 406 \\
LSM, DNS & 30 & 8163 & 1180 & 1119 & 772 & 953 & 1271 & 891 & 976 & 1001 \\
VLSM, PIV & 5120 & 5120 & 1055 & 885 & 384 & 359 & 861 & 915 & 314 & 347 \\
VLSM, DNS & 20 & 5529 & 748 & 556 & 587 & 854 & 764 & 557 & 642 & 821 \\
\hline \hline
\end{tabular}

\section{RESULTS}

\section{A. Instantaneous visualization of filtered velocity field}

The filtered, instantaneous streamwise velocity field from the computational data set is visualized at one snapshot over a small range of spanwise locations $z / \delta=0.3-0.8$ in Fig. 5 [46]. The fluctuating large-scale streamwise velocity field is shown in a streamwise-wall-normal plane in red and blue contours, while the mean-added velocity field is shown at a single value $U_{L} / U_{\infty}=0.85$ in an isosurface in green. The value of the $U_{L}$ isosurface was chosen to match the convection speed of the large-scale flow, which was calculated using a cross correlation between two consecutive snapshots. This $U_{L}$ isosurface is not collocated with the intermittent edge of the boundary layer. The small-scale streamwise velocity field is shown using isosurfaces at two values $u_{s}\left(x, y, z, t_{0}\right) / U_{\infty}= \pm 0.06$ in black and white.

The small-scale isosurfaces fully populate the space near the wall in Fig. 5, where small-scale fluctuations exceed the isocontour threshold. Farther from the wall, above $y / \delta \approx 0.25$, the smallscale fluctuations are sparse when the fluctuating large-scale streamwise velocity field is positive and dense when the fluctuating large-scale streamwise velocity field is negative, consistent with a sweep and ejection representation of the flow [18]. The threshold chosen to visualize the small-scale field affected at what heights the isosurfaces appeared, but did not affect the qualitative behavior of the results until the isosurfaces fully filled the space or restricted the isosurfaces to lie only below $y / \delta \approx 0.1$.

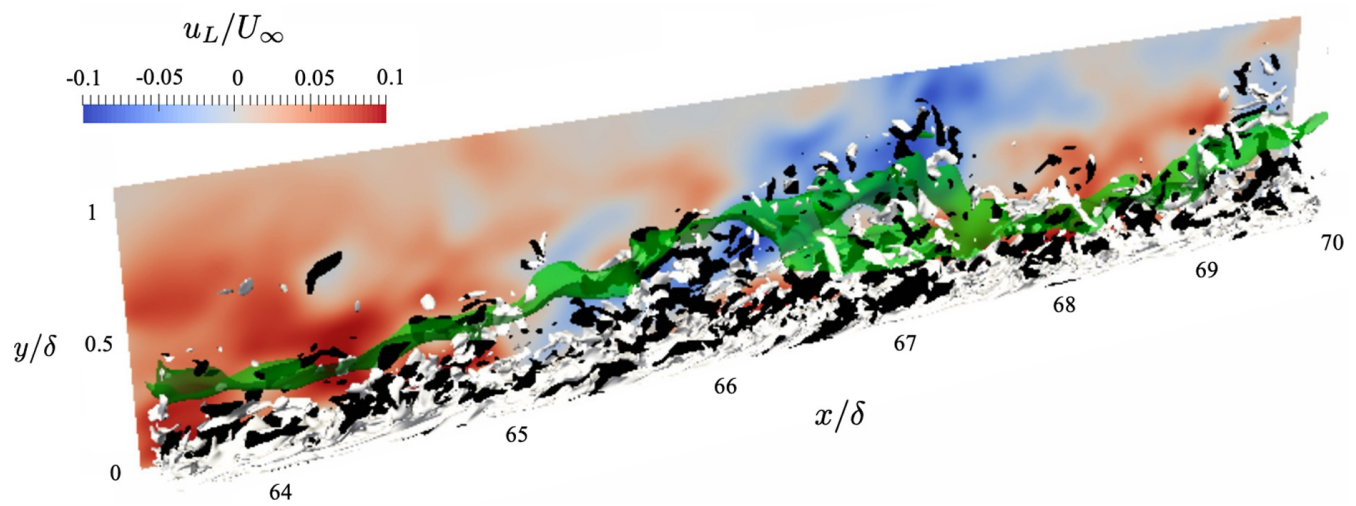

FIG. 5. Visualization of the instantaneous, filtered streamwise velocity field of a turbulent boundary layer [46]. Red and blue contours represent the large-scale fluctuation $u_{L}$. The white and black isosurfaces represent the small-scale fluctuation $u_{s}$ at values of $\pm 0.06 U_{\infty}$. The green isosurface represents an isosurface of the large-scale laboratory-frame streamwise velocity field $U_{L}$ at a value of $0.85 U_{\infty}$, which is the approximated convection velocity of the large scale in this snapshot. 


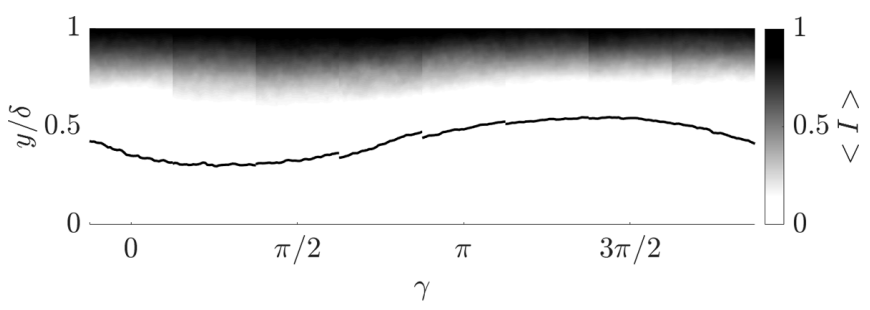

FIG. 6. Conditional projection average of the intermittency variable $I(x, y, t)$ based on a projection onto a model of a LSM. The isocontour $\langle u\rangle+\bar{U}=0.8 U_{\infty}$ is plotted for reference.

The rise and fall of the mean-added large-scale streamwise velocity isosurface (green in Fig. 5) follows a similar trend as the small-scale isosurfaces. The visual similarity between the height at which the green isosurface is observed and the height where small scales are active suggests a hypothesis that small-scale activity may be consistently present along lines of constant value of the local large-scale flow. This hypothesis is consistent with previous observations that isocontours of the full velocity field coincide with the locations of shear layers $[12,47]$ and may be related to the concept of critical layers, which amplify small-scale perturbations where the perturbation speed matches the speed of the base flow or mean flow [48]. Because of this observation, the mean-added large-scale velocity isocontour is visualized in addition to the fluctuating large-scale velocity field in the results that follow.

\section{B. Results for conditional projection averaging}

An intermittency variable $I$ was defined with the same definition used by Chauhan et al. [49] such that its value was zero within the boundary layer and one outside of it, with a sharp interface indicating the instantaneous height of the turbulent-non-turbulent interface (TNTI) for each frame of PIV. The intermittency variable $I(x, y, t)$ was averaged using the conditional projection averaging technique with a LSM model, setting $q=I$ using Eq. (9). Figure 6 shows the result of this averaging process. The averaged streamwise velocity field $\langle U\rangle$ was also computed and an isocontour of $\langle U\rangle=$ 0.8 was overlaid, highlighting that this isocontour lies within the boundary layer height $\delta$ on average.

The velocity fields and intensities averaged using the CPA method are shown in Figs. 7 and 8. Results conditioned on a projection with a LSM model in the wake region are shown in Fig. 7, while results conditioned on a projection with a VLSM model in the logarithmic region are shown in Fig. 8. Results averaged around the approximated phase of LSMs are shown with a linear wallnormal scaling, while the results averaged around the approximated phase of VLSMs are shown with a logarithmic wall-normal scaling. A single isocontour of the average streamwise velocity field summed with the mean $\langle U\rangle(x, y)=\langle u\rangle(x, y)+\bar{U}(x, y)=c$ is overlaid on each panel of Figs. 7 and 8, with $c=0.8 U_{\infty}$ in Fig. 7 and $c=0.6 U_{\infty}$ in Fig. 8, corresponding to the wave speed of the LSM and VLSM models, respectively. The visualization of the isocontour was motivated by the spatial relationship between the mean-added streamwise velocity field and the small-scale intensity that was instantaneously observed in Fig. 5. The filter sizes for the LSM- and VLSM-averaged small-scale velocity fields were $\sigma_{G}=0.5 \delta$ and $\sigma_{G}=0.125 \delta$, respectively. Experimental data were used to compute the results of Figs. 7(a)-7(d), 8(b), and 8(c), while computational data were used to compute the results of Figs. 7(e), 8(a), 8(d), and 8(e). Experimental data were used when possible due to the larger number of temporal snapshots yielding better convergence, while computational data were used for all averages of the spanwise velocity and to resolve near-wall behaviors.

The averaged unfiltered streamwise velocity fields [Figs. 7(a) and 8(a)] yielded periodic structures with a period and wall-normal coherence matching those of the model used for projection. This behavior was expected and was plotted to provide a reference for the local large-scale behavior when considering the small-scale velocity intensities. The small-scale velocity intensities averaged 


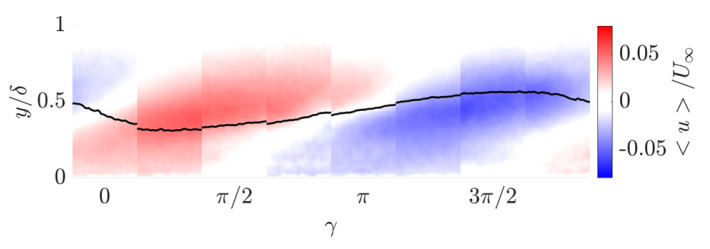

(a)

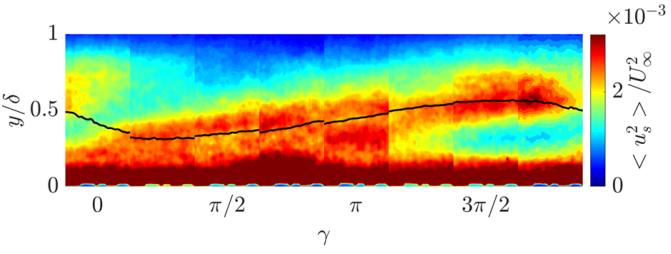

(b)

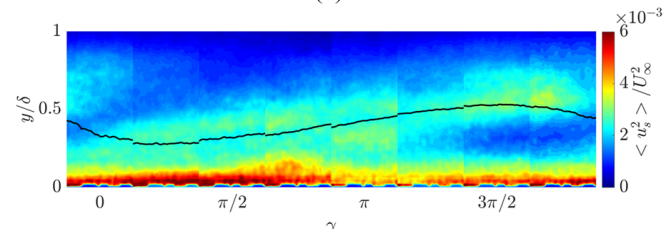

(d)

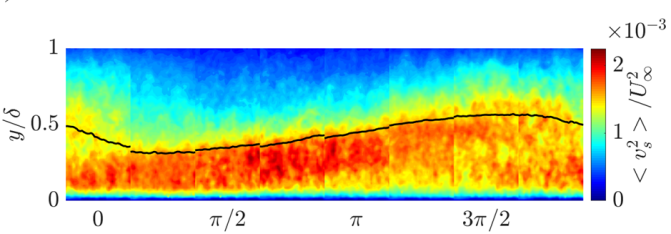

(c)

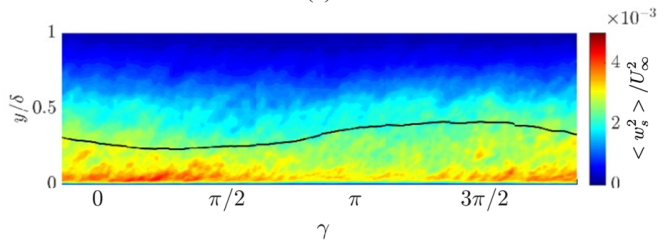

(e)

FIG. 7. (a) Streamwise velocity field and (b)-(e) small-scale velocity intensities averaged using projection onto the LSM model. Conditional small-scale (b) and (d) streamwise, (c) wall-normal, and (e) spanwise intensities are shown. Results are derived from (a)-(d) experimental and (e) numerical data.

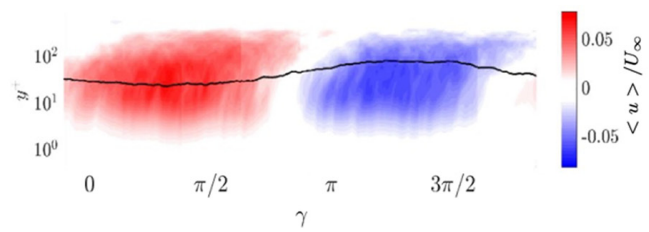

(a)

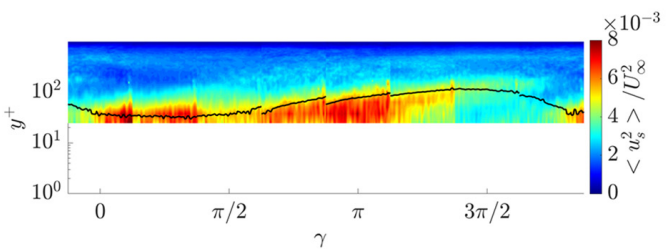

(b)

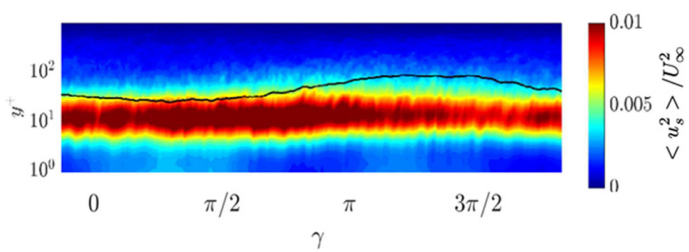

(d)

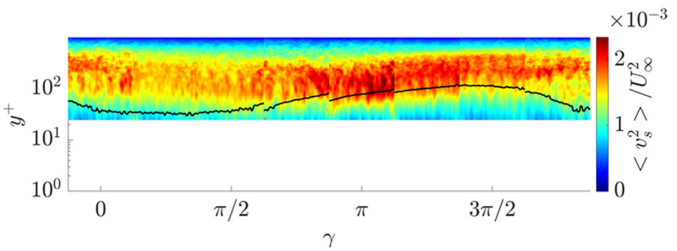

(c)

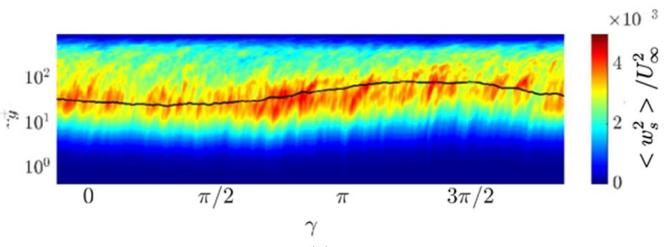

(e)

FIG. 8. (a) Streamwise velocity field and (b)-(e) small-scale velocity intensities averaged using projection onto the VLSM model. Conditional small-scale (b) and (d) streamwise, (c) wall-normal, and (e) spanwise intensities are shown. Results are derived from (b) and (c) experimental and (a), (d), and (e) numerical data. 
on the phase of LSMs [Figs. 7(b)-7(e)] were stronger farther from the wall in phases in which the averaged large-scale streamwise velocity fluctuation $\langle u\rangle$ was negative and stronger nearer to the wall in phases in which $\langle u\rangle$ was positive, consistent with previous studies [20]. All three velocity intensities showed a clear dropoff in intensity at the averaged TNTI location. Within the internal structure of the boundary layer, the streamwise and wall-normal small-scale intensities showed an additional localization that was not observed in the spanwise small-scale intensity. The color bars were chosen to highlight the wake region localization observed for the streamwise and wall-normal intensities in Figs. 7(b) and 7(c), while the color bars for the streamwise velocity in Fig. 7(d) and for the spanwise velocity in Fig. 7(e) were chosen to highlight behavior in the logarithmic region.

The streamwise velocity intensity in the wake region of Fig. 7 (b) $(y / \delta \geqslant 0.1)$ had a roughly constant maximum amplitude in the wake region across the large-scale phases at a range of heights $0.4 \leqslant y / \delta \leqslant 0.6$. The region of constant amplitude overlapped with the isocontour $\langle U\rangle / U_{\infty}=0.8$. The wall-normal small-scale velocity intensity in Fig. 7(c) was less peaked and less consistent in maximum amplitude than the streamwise intensity but still showed a dropoff in intensity below the isocontour $\langle U\rangle / U_{\infty}=0.8$, rather than at the edge of the boundary layer, at heights that varied with the large-scale phase. The streamwise velocity intensity [Fig. 7(d)] and spanwise velocity intensity [Fig. 7(e)] were plotted with color bars that highlighted their behavior in the logarithmic region, while still being averaged on the LSM model in the wake region. Their behavior in the logarithmic region shows change in the maximum amplitude over the large-scale phases for both velocity components. The height at which the maximum occurs appears fairly constant across the large-scale phases.

The analysis was performed again with a projection on a VLSM model in the logarithmic region. The streamwise velocity in the logarithmic region, shown in Fig. 8(b), showed variation in amplitude as well as a change in the height where that maximum occurred. The maximum height approximately followed the isocontour $\langle U\rangle / U_{\infty}=0.6$. The wall-normal small-scale velocity intensity [Fig. 8(c)] was most energetic in the wake region at a fairly constant height across the VLSM phases. Variation in the maximum amplitude was observed, stronger in the presence of a negative VLSM fluctuation and weaker in the phases of a positive VLSM fluctuation. The color bar for the streamwise small-scale velocity intensity was shifted to highlight the near-wall cycle in Fig. 8(d). The maximum amplitude of the streamwise small-scale velocity intensity varied over the VLSM phases and remained at a fairly constant wall-normal location. The spanwise velocity intensity [Fig. 8(e)] had fairly constant maximum amplitude across the VLSM phases. The height at which the maximum occurred varied across the phases and coincided with the height of the $\langle U\rangle / U_{\infty}=0.6$ isocontour that was overlaid (the assumed wave speed of the VLSM model).

\section{DISCUSSION AND CONCLUSIONS}

Both height and amplitude variation effects were observed in the small-scale velocity intensities averaged using the conditional projection averaging technique. The height and amplitude variation trends depended on the component of the small-scale velocity field being averaged, the wall-normal region where the small-scale activity was studied, and the type of large-scale structure used for projection. In the wake, $u_{s}$ and $v_{s}$ both showed some evidence of height variation when the projection was performed with the LSM model: Both intensity fields showed localization and a change in the wall-normal location of the maximum amplitude over the phases that was not exclusively tied to the location of the TNTI [Figs. 7(b) and 7(c)]; however, when $v_{s}$ was averaged using the VLSM model for projection in Fig. 8(c), amplitude variation was observed with little height variation. In the logarithmic region, $u_{s}$ and $w_{s}$ showed evidence of amplitude variation when the projection applied used the LSM model in the wake, but both showed evidence of height variation when the projection applied used the VLSM model in the logarithmic region. Further, $u_{s}$ in the near-wall cycle region showed evidence of amplitude variation when the projection applied used the VLSM model in the logarithmic region. 


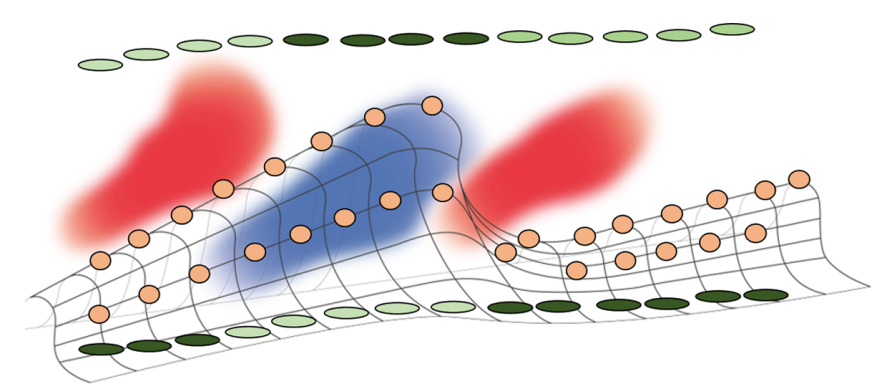

FIG. 9. Interpretation of the height and amplitude variation behavior observed. Large-scale fluctuations are shown in red and blue with a large-scale velocity isosurface drawn in gray curves. Small-scale features in the same region of the flow as the large scales, represented with orange circles, follow the large-scale isosurface. Small scales in other regions of the flow, represented with green ovals, change amplitude depending on the local shear stress imposed by the large-scale fluctuations.

A hypothesis to explain these various observations is provided in Fig. 9. Small scales that were active and strong in the same region of the flow as the large-scale structure used for the projection often showed evidence of height variation $\left(u_{s}\right.$ and $v_{s}$ in the wake when averaged on LSMs and $u_{s}$ and $w_{s}$ in the logarithmic region when averaged on VLSMs). Small scales that were active and strong in a different region of the flow as the large-scale structure used for the condition projection often showed evidence of amplitude variation $\left(u_{s}\right.$ and $w_{s}$ in the logarithmic region when averaged on LSMs, $v_{s}$ in the wake when averaged on VLSMs, and $u_{s}$ in the near-wall cycle region when averaged on VLSMs). We hypothesize that small scales that are physically near the central height of a large-scale coherent structure experience height variation as a result of that structures' presence (through participation in packets and/or bursts and sweeps), while small scales that physically occupy a different space than the large-scale coherent structure of interest instead experience a change in the stress that they experience [22,50]. A faster (slower) structure above the small scale yields a stronger (weaker) local shear stress for that small scale, while a faster (slower) structure below the small scale yields a weaker (stronger) local shear stress for that small scale. In Fig. 9, red and blue objects represent the large-scale feature, orange represents the small scales in the same region of the flow as the large scale, and green represents the small scales in other regions of the flow. Darker (lighter) green indicates stronger (weaker) small scales. A black isosurface of the large scale including the mean is also provided, and the orange small scales are shown following that isosurface.

The height variation behavior observed here is consistent with the preferred spatial organization of vortical structure observed by, e.g., Adrian [51] and the vortical fissures of Klewicki and Hirschi [52], as well as the burst and sweep perspective of Kline et al. [53]. The stress-based amplitude modulation behavior observed here is consistent with the observations of Baars et al. [22]. In this paper, we connect the statistical observations characterized by the amplitude modulation statistic and the structural picture associated with vortex clusters and hairpin packets. Investigations of causal and dynamic interactions between the large- and small-scale motions are reserved for future work.

Previously, no significant differences were observed in the correlations between large-scale streamwise velocity signals and the envelope of different small-scale velocity components $u_{s}, v_{s}$, and $w_{s}$ [25]. However, in the present work, the height versus amplitude variation trend seems dependent on the component of the small-scale velocity being considered. The reason for this difference may be that the present study did not normalize by the average small-scale intensity locally. It was important in this study to observe the intensity level normalized by a global parameter, in order to track the maximum value of the intensity across the height of the boundary layer. By retaining the strengths of different components of the velocity field at different heights in the flow, the strongest 
small-scale structures were highlighted and continuity of the amplitude of the small-scale structures was observed.

The organization of the small scales that occupy the same space as the large-scale feature of interest along a specific isocontour of the large-scale feature is not conclusively shown in this work, but is suggested by it. Such a localization may suggest that small scales sit at a constant height in some local definition of the large-scale flow, rather than at a constant height relative to a mean. Critical layer mechanisms are responsible for the amplification of structures at a specific height relative to a base flow or a mean [41]. Continued work is suggested to explore whether critical layer mechanisms could be responsible for the amplification of small scales along specific isocontours of some local "large-scale flow" in fully developed turbulent boundary layers.

\section{ACKNOWLEDGMENTS}

This work was made possible through United States Air Force Grants No. FA9550-12-1-0060 and No. FA9550-16-1-0361 and through a National Defense Science and Engineering Graduate Fellowship. The authors would like to thank Xiaohua Wu for providing access to and support using his computational data. The support of the Center for Turbulence Research at Stanford University and useful conversations there with Kevin Rosenberg and Aaron Towne are also gratefully acknowledged.

\section{APPENDIX: SENSITIVITY OF RESULTS}

The sensitivity of the results to the details of the projection and averaging technique were examined. Parameters that could have an effect on the results and were examined were the use of a one-dimensional versus two-dimensional projection, the size of the filter that defined large versus small scales, and the wave speed of the model used for projection. The sensitivity of the results to each of these parameters was explored for the small-scale streamwise velocity intensity averaged on the LSMs in the wake region as a somewhat concise demonstration of the method's sensitivity.

\section{Two-dimensional vs one-dimensional model}

The results were not highly sensitive to the use of a one-dimensional versus a two-dimensional model of the large-scale coherent structure for the projection. The small-scale streamwise velocity intensity averaged on the approximated phase of a LSM, computed using a one-dimensional projection, is shown in Fig. 10(a) and can be compared to Fig. 7(b). Due to the low sensitivity and the improved speed of the method using the one-dimensional projection, the other sensitivity results also used the one-dimensional projection.

\section{Filter width}

The small-scale streamwise velocity intensity defined using different sizes of a Gaussian filter are shown in Fig. 10. The definition of the small scales $u_{s}$ was modified by changing the definition of the standard deviation of the Gaussian filter $\sigma_{G}$. The filter width was quartered to $0.125 \delta$ [Fig. 10(b)] and doubled to $1 \delta$ [Fig. 10(c)]. The color bar was adjusted to enable the visualization of each result. The size of the filter was observed to affect the magnitude of the intensity of the small scales, but not to alter the spatial organization of the intensity. The effect of the spatial resolution was also studied by filtering the smallest scales and was also found to affect the magnitude of the intensity of the small scales, but not to alter the qualitative behavior.

\section{Projection model wave speed}

The wave speed of the model used for projection was varied in order to adjust the height where projection was performed. The height of projection was the location where the mean velocity profile 


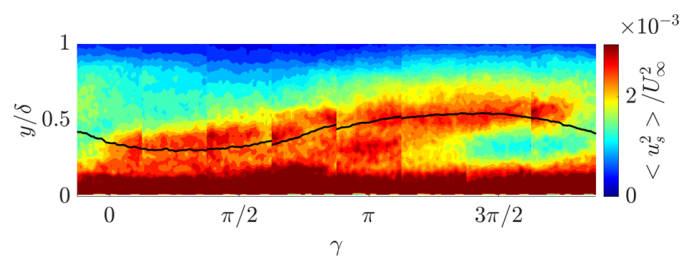

(a)

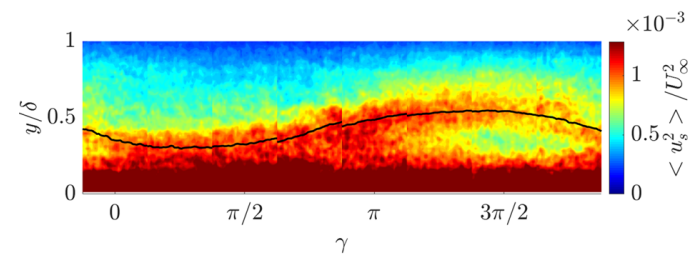

(b)

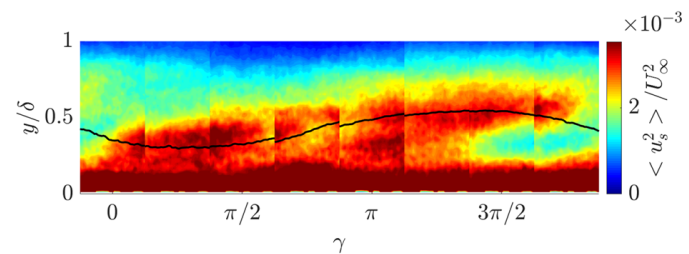

(c)

FIG. 10. Averaged streamwise small-scale velocity intensities are shown in the streamwise-wall-normal plane using the experimental data and the one-dimensional conditional projection averaging approach using a LSM model. The small scales are defined using a standard deviation of (a) $0.5 \delta$, (b) $0.125 \delta$, and (c) $1 \delta$ for the Gaussian filter.

matched the model wave speed: $\bar{U}\left(y_{0}\right)=c$ where $c$ was changed to $0.7 U_{\infty}$ and $0.9 U_{\infty}$ (results in the study used $0.6 U_{\infty}$ and $\left.0.8 U_{\infty}\right)$.

With the change of the projection height, the structures in the averaged streamwise velocity field [Figs. 11(a) and 11(b)] shifted towards and away from the wall, respectively. In each panel the isocontour corresponding to $\langle u\rangle+\bar{U}=c$ of the large scale is shown as a solid black curve. Figure 11 demonstrates that the streamwise small-scale velocity intensity was sensitive to the height where the condition projection took place. In the $c=0.7 U_{\infty}$ case [Fig. 11(c)], amplitude variation was observed more strongly in the small-scale streamwise velocity intensity in the wake region, while height variation was observed in the logarithmic region. This may have reflected that the phases of the VLSMs were being averaged as much as or more than the phase of the LSMSs, yielding height variation in the logarithmic region around the VLSMs, rather than in the wake

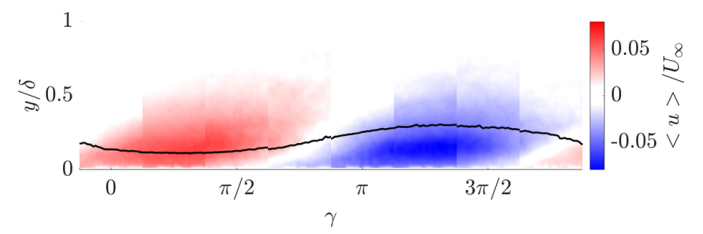

(a)

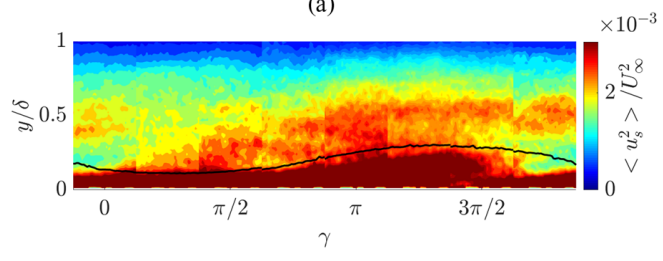

(c)

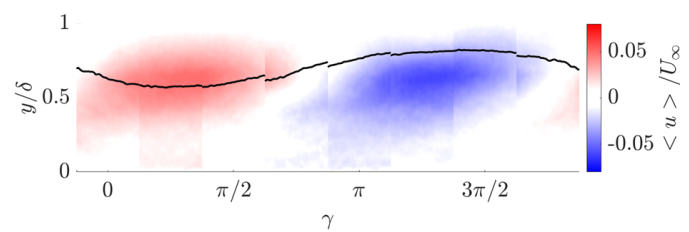

(b)

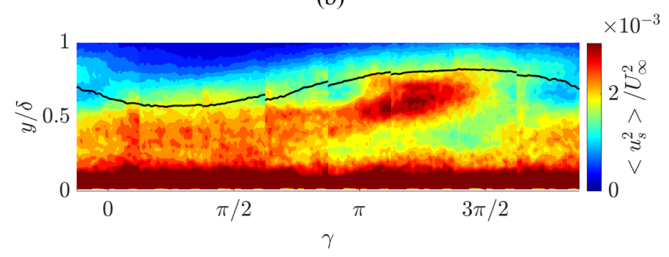

(d)

FIG. 11. Phase-averaged PIV data with an assumed large-scale convection velocity of (a) and (c) $0.7 U_{\infty}$ and (b) and (d) $0.9 U_{\infty}$. Conditional projection averages are shown of (a) and (b) the streamwise fluctuating velocity field and (c) and (d) the streamwise small-scale velocity intensity. The isocontour $\langle U\rangle=c$ is shown by a solid black line in each panel. 
region around the LSMs. This result suggests that the height of projection has a leading-order effect on which large-scale coherent structure is averaged upon. In the $c=0.9 U_{\infty}$ case [Fig. 11(d)], some height variation was observed but was much less localized or strong than was observed for the original results in Fig. 7. The localization of the small-scale streamwise velocity intensity that was still observed was not isolated along the curve $\langle U\rangle=0.9 U_{\infty}$, but instead showed a height profile associated more with the location where $\langle U\rangle=0.8 U_{\infty}$ would pass.

[1] S. K. Robinson, Coherent motions in the turbulent boundary layer, Annu. Rev. Fluid Mech. 23, 601 (1991).

[2] K. C. Kim and R. J. Adrian, Very large-scale motion in the outer layer, Phys. Fluids 11, 417 (1999).

[3] M. Guala, S. Hommema, and R. Adrian, Large-scale and very-large-scale motions in turbulent pipe flow, J. Fluid Mech. 554, 521 (2006).

[4] A. Lozano-Durán, O. Flores, and J. Jiménez, The three-dimensional structure of momentum transfer in turbulent channels, J. Fluid Mech. 694, 100 (2012).

[5] R. J. Adrian, C. D. Meinhart, and C. D. Tomkins, Vortex organization in the outer region of the turbulent boundary layer, J. Fluid Mech. 422, 1 (2000).

[6] J. P. Monty, N. Hutchins, H. C. H. Ng, I. Marusic, and M. S. Chong, A comparison of turbulent pipe, channel and boundary layer flows, J. Fluid Mech. 632, 431 (2009).

[7] C. Smith and S. Metzler, The characteristics of low-speed streaks in the near-wall region of a turbulent boundary layer, J. Fluid Mech. 129, 27 (1983).

[8] T. Theodorsen, Proceedings of the Midwestern Conference on Fluid Mechanics (Ohio State University, Columbus, 1952).

[9] R. E. Falco, Coherent motions in the outer region of turbulent boundary layers, Phys. Fluids 20, S124 (1977).

[10] C. H. P. Chen and R. F. Blackwelder, Large-scale motion in a turbulent boundary layer: A study using temperature contamination, J. Fluid Mech. 89, 1 (1978).

[11] C. D. Meinhart and R. J. Adrian, On the existence of uniform momentum zones in a turbulent boundary layer, Phys. Fluids 7, 694 (1995).

[12] J. Eisma, J. Westerweel, G. Ooms, and G. E. Elsinga, Interfaces and internal layers in a turbulent boundary layer, Phys. Fluids 27, 055103 (2015).

[13] T. Saxton-Fox and B. McKeon, Coherent structures, uniform momentum zones and the streamwise energy spectrum in wall-bounded turbulent flows, J. Fluid Mech. 826, R6 (2017).

[14] J. Jiménez, S. Hoyas, M. P. Simens, and Y. Mizuno, Turbulent boundary layers and channels at moderate Reynolds numbers, J. Fluid Mech. 657, 335 (2010).

[15] G. Eitel-Amor, R. Örlü, P. Schlatter, and O. Flores, Hairpin vortices in turbulent boundary layers, Phys. Fluids 27, 025108 (2015).

[16] J. Jiménez, Cascades in wall-bounded turbulence, Annu. Rev. Fluid Mech. 44, 27 (2012).

[17] L. S. G. Kovasznay, V. Kibens, and R. F. Blackwelder, Large-scale motion in the intermittent region of a turbulent boundary layer, J. Fluid Mech. 41, 283 (1970).

[18] H. Nakagawa and I. Nezu, Structure of space-time correlations of bursting phenomena in an open-channel flow, J. Fluid Mech. 104, 1 (1981).

[19] N. Hutchins and I. Marusic, Large-scale influences in near-wall turbulence, Philos. Trans. R. Soc. A 365, 647 (2007).

[20] D. Chung and B. J. McKeon, Large-eddy simulation of large-scale structures in long channel flow, J. Fluid Mech. 661, 341 (2010).

[21] M. F. Howland and X. I. A. Yang, Dependence of small-scale energetics on large scales in turbulent flows, J. Fluid Mech. 852, 641 (2018).

[22] W. J. Baars, N. Hutchins, and I. Marusic, Reynolds number trend of hierarchies and scale interactions in turbulent boundary layers, Philos. Trans. R. Soc. A 375, 20160077 (2017). 
[23] R. Mathis, J. P. Monty, N. Hutchins, and I. Marusic, Comparison of large-scale amplitude modulation in turbulent boundary layers, pipes, and channel flows, Phys. Fluids 21, 111703 (2009).

[24] Z. Harun, J. P. Monty, R. Mathis, and I. Marusic, Pressure gradient effects on the large-scale structure of turbulent boundary layers, J. Fluid Mech. 715, 477 (2013).

[25] K. M. Talluru, R. Baidya, N. Hutchins, and I. Marusic, Amplitude modulation of all three velocity components in turbulent boundary layers, J. Fluid Mech. 746, R1 (2014).

[26] T. Kim, G. Blois, J. L. Best, and K. T. Christensen, Experimental evidence of amplitude modulation in permeable-wall turbulence, J. Fluid Mech. 887, A3 (2020).

[27] B. Ganapathisubramani, N. Hutchins, J. P. Monty, D. Chung, and I. Marusic, Amplitude and frequency modulation in wall turbulence, J. Fluid Mech. 712, 61 (2012).

[28] T. Saxton-Fox, Coherent structures, their interactions, and their effects on passive scalar transport and aero-optic distortion in a turbulent boundary layer, Ph.D. thesis, California Institute of Technology, 2018.

[29] X. Wu, P. Moin, and J.-P. Hickey, Boundary layer bypass transition, Phys. Fluids 26, 091104 (2014).

[30] T. Saxton-Fox, B. J. McKeon, and S. Gordeyev, Effect of coherent structures on aero-optic distortion in a turbulent boundary layer, AIAA J. 57, 2828 (2019).

[31] X. Wu, P. Moin, J. M. Wallace, J. Skarda, A. Lozano-Durán, and J.-P. Hickey, Transitional-turbulent spots and turbulent-turbulent spots in boundary layers, Proc. Natl. Acad. Sci. USA 114, E5292 (2017).

[32] F. H. Clauser, in Advances in Applied Mechanics, edited by H. L. Dryden and T. von Kármán (Elsevier, Amsterdam, 1956), Vol. 4, pp. 1-51.

[33] H. Fernholz and P. Finley, The incompressible zero-pressure-gradient turbulent boundary layer: An assessment of the data, Prog. Aerosp. Sci. 32, 245 (1996).

[34] D. B. DeGraaff and J. K. Eaton, Reynolds-number scaling of the flat-plate turbulent boundary layer, J. Fluid Mech. 422, 319 (2000).

[35] D. Marr and E. Hildreth, Theory of edge detection, Proc. R. Soc. London Ser. B 207, 187 (1980).

[36] T. Lindeberg, Scale-space theory: A basic tool for analysing structures at different scales, J. Appl. Stat. 21, 225 (1994).

[37] R. Mathis, N. Hutchins, and I. Marusic, Large-scale amplitude modulation of the small-scale structures in turbulent boundary layers, J. Fluid Mech. 628, 311 (2009).

[38] C. M. de Silva, D. Chandran, R. Baidya, N. Hutchins, and I. Marusic, Periodicity of large-scale coherence in turbulent boundary layers, Int. J. Heat Fluid Flow 83, 108575 (2020).

[39] I. Jacobi and B. J. McKeon, Phase relationships between large and small scales in the turbulent boundary layer, Exp. Fluids 54, 1481 (2013).

[40] B. J. McKeon, The engine behind (wall) turbulence: Perspectives on scale interactions, J. Fluid Mech. 817, P1 (2017).

[41] B. J. McKeon and A. S. Sharma, A critical-layer framework for turbulent pipe flow, J. Fluid Mech. 658, 336 (2010).

[42] P. J. Schmid, Dynamic mode decomposition of numerical and experimental data, J. Fluid Mech. 656, 5 (2010).

[43] A. Towne, O. T. Schmidt, and T. Colonius, Spectral proper orthogonal decomposition and its relationship to dynamic mode decomposition and resolvent analysis, J. Fluid Mech. 847, 821 (2018).

[44] O. T. Schmidt and P. J. Schmid, A conditional space-time POD formalism for intermittent and rare events: Example of acoustic bursts in turbulent jets, J. Fluid Mech. 867, R2 (2019).

[45] R. F. Blackwelder and R. E. Kaplan, On the wall structure of the turbulent boundary layer, J. Fluid Mech. 76, 89 (1976).

[46] K. Rosenberg, T. Saxton-Fox, A. Lozano-Durán, A. Towne, and B. J. McKeon, Proceedings of the Summer Program 2016 (Center for Turbulence Research, Stanford, 2016), p. 305.

[47] C. M. de Silva, N. Hutchins, and I. Marusic, Uniform momentum zones in turbulent boundary layers, J. Fluid Mech. 786, 309 (2016).

[48] P. G. Drazin and W. H. Reid, Hydrodynamic Stability (Cambridge University Press, Cambridge, 2004).

[49] K. Chauhan, J. Philip, C. M. de Silva, N. Hutchins, and I. Marusic, The turbulent/non-turbulent interface and entrainment in a boundary layer, J. Fluid Mech. 742, 119 (2014). 
[50] C. Zhang and S. I. Chernyshenko, Quasisteady quasihomogeneous description of the scale interactions in near-wall turbulence, Phys. Rev. Fluids 1, 014401 (2016).

[51] R. J. Adrian, Hairpin vortex organization in wall turbulence, Phys. Fluids 19, 041301 (2007).

[52] J. C. Klewicki and C. R. Hirschi, Flow field properties local to near-wall shear layers in a low Reynolds number turbulent boundary layer, Phys. Fluids 16, 4163 (2004).

[53] S. J. Kline, W. C. Reynolds, F. A. Schraub, and P. W. Runstadler, The structure of turbulent boundary layers, J. Fluid Mech. 30, 741 (1967). 